\title{
Crystal structure and versatile functional roles of the COP9 signalosome subunit 1
}

\author{
Jung-Hoon Lee ${ }^{a, 1}$, Lina $\mathrm{Yi}^{\mathrm{a}, \mathrm{b}, 1}$, Jixi Li ${ }^{\mathrm{a}, \mathrm{b}}$, Katrin Schweitzerc, Marc Borgmann ${ }^{c}$, Michael Naumann ${ }^{c}$, and Hao Wi, ${ }^{\mathrm{a}, \mathrm{b}, 2}$ \\ ${ }^{a}$ Department of Biochemistry, Weill Cornell Medical College, New York, NY 10065; ${ }^{b}$ Program in Cellular and Molecular Medicine, Children's Hospital Boston \\ and Department of Biological Chemistry and Molecular Pharmacology, Harvard Medical School, Boston, MA 02115; and 'Institute of Experimental Internal \\ Medicine, Otto-von-Guericke Universität Magdeburg, 39120 Magdeburg, Germany
}

Edited by Wolfgang Baumeister, Max-Planck-Institute of Biochemistry, Martinsried, Germany, and approved June 10, 2013 (received for review February 17, 2013)

The constitutive photomorphogenesis 9 (COP9) signalosome (CSN) plays key roles in many biological processes, such as repression of photomorphogenesis in plants and protein subcellular localization, DNA-damage response, and NF- $\kappa$ B activation in mammals. It is an evolutionarily conserved eight-protein complex with subunits CSN1 to CSN8 named following the descending order of molecular weights. Here, we report the crystal structure of the largest CSN subunit, CSN1 from Arabidopsis thaliana (atCSN1), which belongs to the Proteasome, COP9 signalosome, Initiation factor 3 (PCI) domain containing CSN subunit family, at $2.7 \AA$ Å resolution. In contrast to previous predictions and distinct from the $\mathrm{PCl}$-containing $26 \mathrm{~S}$ proteasome regulatory particle subunit Rpn6 structure, the atCSN1 structure reveals an overall globular fold, with four domains consisting of helical repeat-I, linker helix, helical repeat-II, and the C-terminal PCI domain. Our small-angle X-ray scattering envelope of the CSN1-CSN7 complex agrees with the EM structure of the CSN alone (apo-CSN) and suggests that the PCI end of each molecule may mediate the interaction. Fitting of the CSN1 structure into the CSN-Skp1-Cul1-Fbox (SCF) EM structure shows that the $\mathrm{PCl}$ domain of CSN1 situates at the hub of the CSN for interaction with several other subunits whereas the linker helix and helical repeat-II of CSN1 contacts SCF using a conserved surface patch. Furthermore, we show that, in human, the C-terminal tail of CSN1, a segment not included in our crystal structure, interacts with IкBo in the NF- $\mathrm{B}$ p pathway. Therefore, the CSN complex uses multiple mechanisms to hinder NF-KB activation, a principle likely to hold true for its regulation of many other targets and pathways.

$\mathbf{T}$ he constitutive photomorphogenesis 9 (COP9) signalosome (CSN) is a more than $300-\mathrm{kDa}$ complex that was first identified as a negative regulator of Constitutive Photomorphogenesis (COP) in plants $(1,2)$. In the subsequent years, the highly conserved protein complex was also found in fungi $(3,4)$, Caenorhabditis elegans (5), Drosophila melanogaster (6), and mammals $(7,8)$. The most studied function of the CSN complex in eukaryotes is the regulation of protein degradation through two pathways, deneddylation (9-11) and deubiquitination $(12,13)$. In the deneddylation pathway, the CSN complex can influence the cullin-RING ligase activity by removing Nedd8, a ubiquitin-like protein, from a cullin $(9,14)$. On the other hand, the CSN complex can also suppress cullin activity through recruitment of the deubiquitination enzyme USP15 (12) or Ubp12p, the Schizosaccharomyces pombe ortholog of human USP15 (13). Other functions of the CSN complex identified in mammalian cells include regulating the phosphorylation of ubiquitin-proteasome pathway substrates through CSN-associated kinases (7, 15-18). Overall, the CSN complex appears to be a key player in protein subcellular localization (19, 20), DNA-damage response (21), NF- $\kappa \mathrm{B}$ activation (22), development, and cell cycle control (23, 24). Thus, the functions of the CSN complex are beyond the regulation of light-dependent reaction in plants.

The CSN complex in most of the species contains eight subunits named CSN1 to CSN8, in order of decreasing size. All eight subunits share homologous sequences with "lid" components of the $26 \mathrm{~S}$ proteasome regulatory particle and the eukaryotic translation initiation factor $3(\mathrm{eIF} 3)(7,25)$. Among these eight subunits, CSN6 and catalytic CSN5 contain a conserved MPNdomain (MOV34, Pad1N-terminal) (26), and the rest of the CSN subunits bear a PCI-domain (Proteasome, COP9 signalosome, Initiation factor eIF3). The MPN-domain within CSN5 contains a metal chelating site and forms the catalytic region of the isopeptidase for deneddylation (27). Recently, the crystal structures of the CSN6-MPN domain and the CSN5 subunit have been revealed $(28,29)$. Interestingly, amino acids 97-131, a flexible segment within the CSN5-MPN domain, were proven to be essential in regulating the isopeptidase states of CSN5 (29). PCI is an 200 -amino acid domain, beginning with an N-terminal helical bundle arrangement and ending with a globular winged-helix subdomain $(30,31)$. A number of interactions between PCI domains of CSN subunits have been identified by the yeast twohybrid system $(32,33)$. Dessau et al. reported the crystallographic data of the PCI domain of Arabidopsis thaliana subunit 7, and their in vitro studies also suggested that the PCI domain mediates and stabilizes protein-protein interactions within the complex (34).

Although many speculated on how the CSN subunits interact with each other and come into a functional unit, the architecture of the CSN complex was accessed by electron microscopy (EM) $(35,36)$ and native mass spectrometry approaches $(37)$. These studies confirmed structural similarities among CSN, the proteasome lid, and eIF3. Furthermore, the CSN appears to contain two dominant subcomplexes, CSN1/2/3/8 and CSN 4/5/6/7 (37), which correspond to the large and the small segments, respectively, in an EM study of the CSN alone (apo-CSN) (36). An EM study of the CSN in complex with an Skp1-Cul1-Fbox (SCF) E3 ligase was also reported, showing reciprocal regulation between CSN and SCF (38). To date, unfortunately, there is no high-resolution mapping on these subunit interactions.

To further define the CSN structure and to study its functional significance, we feel the need to obtain structures of CSN subunits at an atomic level. In our study, we used Arabidopsis thaliana CSN1 (atCSN1) as a guide to facilitate our understandings of the PCIcontaining CSN subunits. The atCSN1, encoded in the chromosome 3, has 441 amino acids that are $45 \%$ identical in sequence to Homo sapiens CSN1. Among all of the subunits of the complex, CSN1 is known to be the longest and to play a crucial role in complex integrity (39-41). Here, we report the crystal structure of

Author contributions: J.-H.L., L.Y., and H.W. designed research; J.-H.L., L.Y., J.L., K.S., and M.B. performed research; J.-H.L., L.Y., M.N., and H.W. analyzed data; and J.-H.L., L.Y., M.N., and H.W. wrote the paper.

The authors declare no conflict of interest.

This article is a PNAS Direct Submission.

Freely available online through the PNAS open access option.

Data deposition: The atomic coordinates and structure factors have been deposited in the Protein Data bank, www.pdb.org (PDB ID code 4LCT).

1J-H.L. and L.Y. contributed equally to the work.

${ }^{2}$ To whom correspondence should be addressed. E-mail: hao.wu@childrens.harvard.edu.

This article contains supporting information online at www.pnas.org/lookup/suppl/doi:10. 1073/pnas.1302418110/-/DCSupplemental. 
A
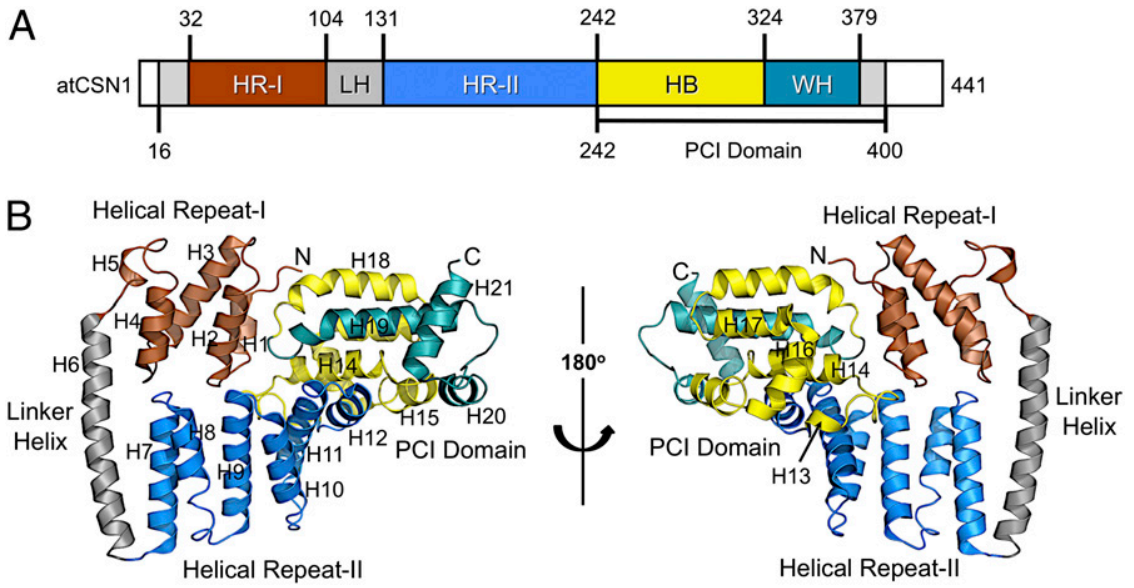

Fig. 1. Crystal Structure of atCSN1. (A) Domain structure of atCSN1. atCSN1 has four major domains, helical repeat-I (HR-I), linker helix (LH), helical repeat-II (HR-II), and PCI Domain (PCID). Similar to other PCIDs, atCSN1 PCID contains the helix-bundle $(\mathrm{HB})$ and winged-helix (WH) subdomains. (B) Ribbon representation of atCSN1, colored by domain structure. Secondary structures are labeled.

atCSN1 and describe its integration within the complex as well as its interaction with $\mathrm{I} \kappa \mathrm{B} \alpha$ in the NF-kB signaling pathway.

\section{Results and Discussion}

Crystallization and Structure Determination. Full-length atCSN1 was first expressed in Escherichia coli BL21 (DE3), but the protein showed partial degradation. Therefore, we performed limited proteolysis on the full-length atCSN1 to identify a stable core fragment. Two Subtilisin-resistant fragments, atCSN1 (16400 aa) and atCSN1 (16-380 aa), identified through mass spectrometry analysis and $\mathrm{N}$-terminal sequencing, appeared to be candidates for downstream crystallization experiments (Fig. S1).
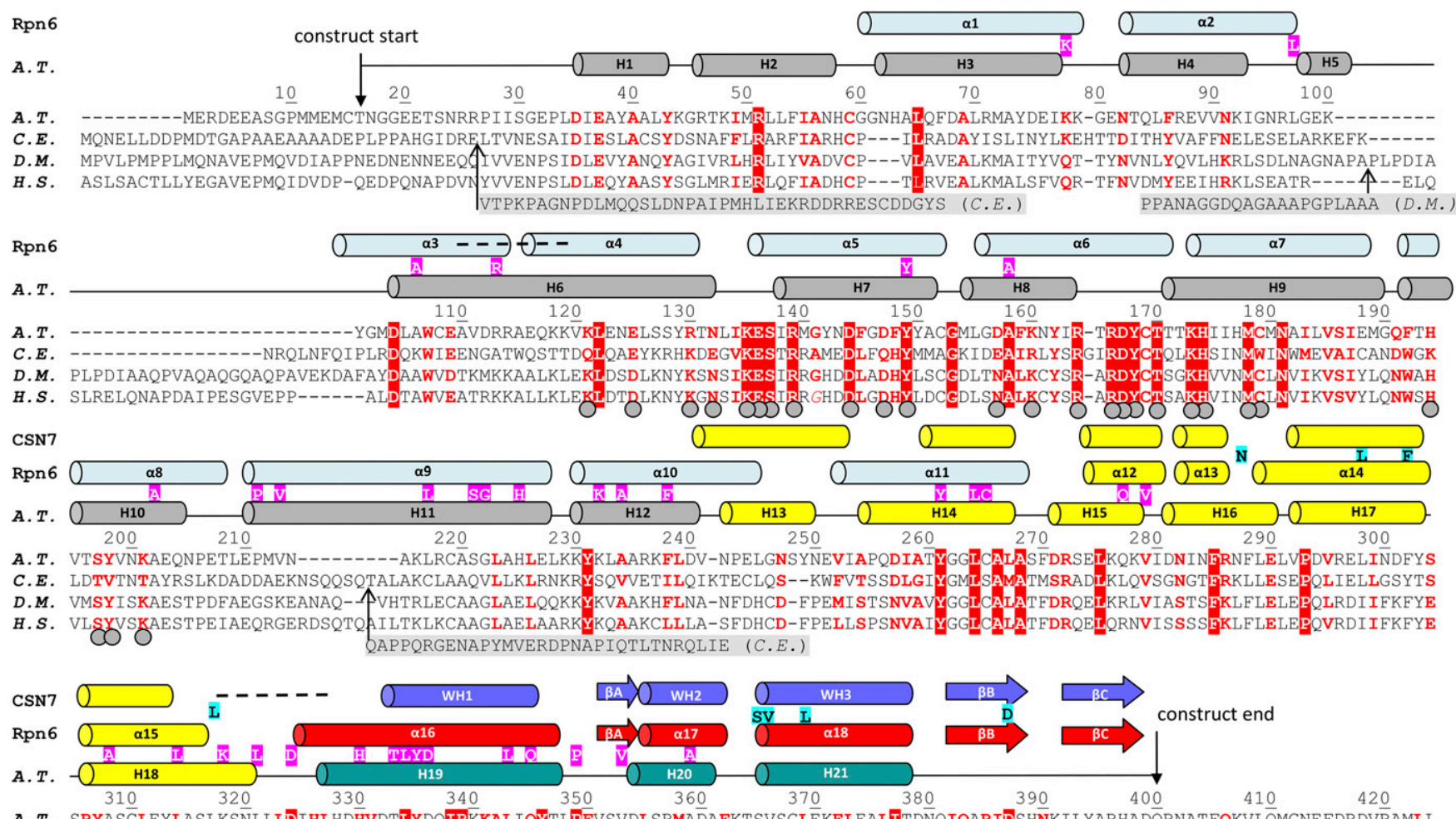

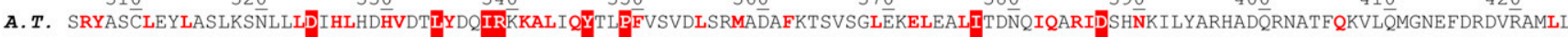
C.E. SRFGRCFE IMRSVKPRLLIDPFISRNVDELFEKIROKCVLQYLOPYSTIKMATMAEAVGMSSAELQLSLLELIEQKHVSLKIDQNEGIVRILDERDENAILKRVNVTCDRATQKAKSLLW

D.M. SKYASCLTLLDEIRDNLLVDMYIAPHVTTLYTKIRNRALIQYFSPYMSADMHKMAMAFNSSVGDLENEVMQLILDGQIQARIDSHNKILFAKEADQRNSTFERALIMGKQYQRHTRMLVI

H.S. SKYASCLKMLDEMKDNLLLDMYLAPHVRTLYTQIRNRALIQYFSPYVSADMHRMAAAFNTTVAALEDELTQLILEGLISARVDSHSKILYARDVDQRSTTFEKSLLMGKEFQRRAKAMML

Fig. 2. Sequence alignment of CSN1 among different species and secondary structure comparisons of atCSN1, Rpn6, and atCSN7 (PCID). Sequence alignment of CSN1 was performed using 10 different species, but we show only the 4 most representative species or most divergent CSN1 sequences in this figure. A.T. (A. Thaliana CSN1); C.E. (C. elegans CSN1); D.M. (D. melanogaster CSN1); H.S. (H. sapiens CSN1). Dash line represents a deletion in the sequence relative to atCSN1. Sequence insertions relative to atCSN1 are in light gray and are labeled with the species names. Strictly conserved residues among the 10 CSN1s are shown in white with a red background; other conserved residues among CSN1s are in red. The secondary structures of atCSN1 HR-I, LH, and HR-II are all presented in gray color whereas HB is in yellow and atCSN1 WH is in deep-teal green. Conserved surface residues within the LH and HR-II, which are possibly involved in SCF interaction, are indicated using gray circles. In addition, the secondary structures of Rpn6 (PDB ID code 3TXM) and atCSN7 (PDB ID code 3CHM) are compared with the secondary structure of atCSN1 side by side $(34,43)$. Rpn6 HB is in yellow, and Rpn6 WH is in red. atCSN7 HB is also in yellow, and atCSN7 WH is in slate blue. Identical residues between atCSN1 and Rpn6 are shown in white with a magenta background. Identical residues between atCSN1 and atCSN7 are shown in black with a cyan background. 
We were able to acquire crystals from the atCSN1 (16-400aa) construct, and the crystal structure was solved by multiwavelength anomalous diffraction at $3.5 \AA$ resolution using crystals derivatized with the $\left[\mathrm{Ta}_{6} \mathrm{Br}_{12}\right]^{2+}$ cluster (Table S1). The final atomic model was refined to $2.7 \AA$ resolution. It contains four atCSN1 molecules, chain A (residues 32-349), chain B (residues 36-379), chain C (residues 32-352), and chain D (residues 32379) (Fig. 1A).

Structure Overview. The crystal structure of atCSN1 consists of two helical repeat domains (HR-I and HR-II), a linker helix (LH), and a PCI domain (PCID) (Fig. 1B). HR-I and HR-II are tandem arrays of two and three helix-turn-helix units respectively. The helix-turn-helix unit is structurally similar to tetratricopeptide repeat (TPR) except that canonical TPR has 34 amino acids within each helix-turn-helix unit (42). Canonical TPR is identified in a wide variety of proteins and usually functions as a protein-protein interaction module (42). LH is the longest single $\alpha$-helix within the protein, and it serves to join together HR-I and HR-II. Similar to other known PCID structures, the PCID of atCSN1 can be divided into two subdomains, helix bundle $(\mathrm{HB})$ subdomain and winged-helix $(\mathrm{WH})$ subdomain $(30$, 34). The overall shape of atCSN1 is like the clubhead of a golf iron with approximate dimensions of $75 \AA \times 57 \AA \times 25 \AA$.

Because the proteasomal lid subunit Rpn6 from $D$. melanogaster (PDB id code 3TXM) shares homologous sequences and PCID with atCSN1 (Fig. 2), we compare the tertiary structures between the two molecules to better understand atCSN1 (Fig. 3A). In Rpn6, the $\alpha$-helical repeats, including a part of the PCID, form a right-handed suprahelical turn (43). The suprahelical fold provides a convex surface for binding of the Rpt6, a "base" subunit of the $26 \mathrm{~S}$ proteasome regulatory particle. In atCSN1, the lengths of the non-PCID helices (H1-H12) are less uniform than the ones in Rpn6 (Fig. 2). Instead of forming an elongated suprahelical structure, the non-PCID helices in atCSN1 emerge into three distinct domains, which closely associate with each other. Meanwhile, the PCID coordinates with both HR-I and HR-II on the other side the molecule. These structural features lead to the formation of an overall compact, globular atCSN1 structure, in contrast to the more extended structure of Rpn6.

PCI Domain of atCSN1. When we put the PCI domains of Rpn6, atCSN7 (34), and atCSN1 side by side, we observe similarities and differences among the three (Fig. $3 A$ and $B$ ). The N-terminal boundary of Rpn6 PCID is different from those of atCSN1 and atCSN7 (31), resulting in four helices in the HB domain of

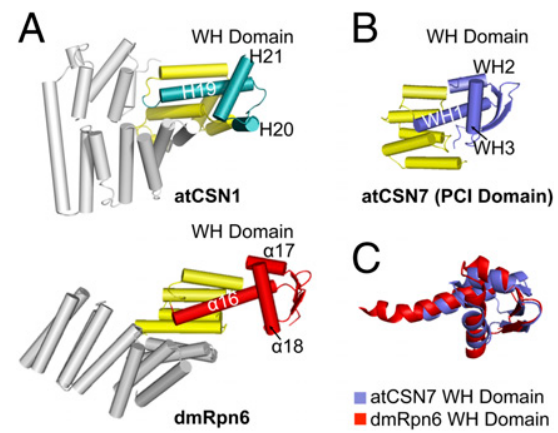

Fig. 3. Tertiary structural comparisons of atCSN1, Rpn6, and atCSN7 (PCID). ( $A$, Upper) Our crystal structure of atCSN1. HB subdomain is in yellow and WH subdomain is in deep-teal green. (Lower) Crystal structure of the proteasomal lid subunit Rpn6 from D. melanogaster (dmRpn6) (PDB ID code 3TXM) (43). Rpn6 HB is in yellow and Rpn6 WH is in red. (B) PCID of atCSN7 (PDB ID code $3 \mathrm{CHM)} \mathrm{(34).} \mathrm{atCSN7} \mathrm{HB} \mathrm{is} \mathrm{in} \mathrm{yellow} \mathrm{and} \mathrm{atCSN7} \mathrm{WH} \mathrm{is} \mathrm{in} \mathrm{slate} \mathrm{blue.} \mathrm{(C)} \mathrm{Su-}$ perposition between Rpn6 WH subdomain and atCSN7 WH subdomain.
Rpn6 PCID and six helices in atCSN1 and atCSN7 (Fig. 2). Whereas Rpn6 WH and atCSN7 WH are well aligned with each other (Fig. 3C), a major structural discrepancy can be observed from atCSN1 WH. Helices $\mathrm{H} 20$ and $\mathrm{H} 21$, as a unit, orient differently in comparison with their counterpart helices in Rpn6 and atCSN7 (Fig. 3). In our atCSN1 crystal structure, we can only visualize a partial WH subdomain, which lacks the last two $\beta$-strands (Figs. 2 and $3 A$ ). One possibility is that the last two $\beta$-strands are not folded as stably as the rest of the atCSN1 structure, as shown by their protease sensitivity (Fig. S1). This instability may be due to the fact that the atCSN1 subunit was crystallized in isolation, rather than in the full CSN complex.

We also contemplated the possibility that the extensive crystal packing around the C-terminal region of the atCSN1 structure (Fig. S2) might have affected the local conformation. Because all known PCI-domain structures share the same fold as in atCSN7 and Rpn6, we attempted to model an alternative and a more complete PCI domain of atCSN1 as a potential conformation within the CSN complex. We first kept all of the current atCSN1 coordinates and added the $\beta \mathrm{B}$ and $\beta \mathrm{C}$ strands of Rpn6 by superimposing Rpn6 $\alpha 17-\alpha 18$ onto atCSN1 H20-H21. However, the modeled $\beta$-sheet crashes into the atCSN1 WH subdomain (Fig. S3A). In both Rpn6 and atCSN7 PCIDs, $\beta$ B and $\beta C$ interact with $\beta \mathrm{A}$ preceding one of the helices in the $\mathrm{WH}$ region (Fig. $\mathrm{S} 3 A$ ). Without $\beta \mathrm{B}$ and $\beta \mathrm{C}$, atCSN1 $\mathrm{H} 20-\mathrm{H} 21$ cannot be held toward the same orientation as the ones in Rpn6 and atCSN7. Therefore, to rebuild the C-terminal end of atCSN1, we aligned Rpn6 $\alpha 16$ with atCSN1 H19 using the conserved residues in these helices (Fig. 2, Fig. S3B) and grafted the rest of the Rpn6 C-terminal structure onto the atCSN1 structure to generate the tentative complete PCID of atCSN1 (Fig. S3C). Interestingly, the intermolecular interactions at the $\mathrm{H} 19-\mathrm{H} 21$ region between chain A and chain B (Fig. S2B) and between chain C and symmetry-related chain D (Fig. S2D) both mimic the intramolecular interaction in the modeled PCI domain, suggesting that the modeled H19-H21 structure cluster represents a conserved interaction in atCSN1 as well.

atCSN1 Interacts with atCSN7 (PCID) Through Its WH Subdomain. It was previously known that CSN1 and CSN7 interact with each other $(32,33)$, and the interaction was quantified using isothermal titration calorimetry, yielding a $K_{\mathrm{D}}$ of $2.2 \pm 0.04 \mu \mathrm{M}$ (34). Given the available crystal structures of atCSN1 and atCSN7, we coexpressed atCSN1 and atCSN7 in E. coli and further investigated this interaction using the small-angle X-ray scattering (SAXS) method. The SAXS patterns of the atCSN1atCSN7 binary complex in solution were recorded to generate the final composite scattering curves (Fig. 4A, Left). By inspecting the Guinier plot of the complex, we saw an absence of protein aggregation and processed the data further. The gyration radius $\left(R_{g}\right)$ of the complex is $32 \AA$ whereas the maximum dimension $\left(D_{\max }\right)$ from the distance distribution function $p(r)$ is $110 \AA$ (Fig. $4 A$, Center). The SAXS envelope of the atCSN1atCSN7 binary complex reconstructed from the scattering patterns appears to be of a tubular shape with one large and one small section (Fig. 4A, Right). The dimensions of the larger section are well consistent with the ones in our atCSN1 crystal structure, and the smaller section has similar dimensions as atCSN7 (PCID). It has been shown that the PCIDs of CSN1 and CSN7 are responsible for the mutual interaction (34), which constrains the PCID of each of the proteins to be near the central part of the SAXS envelope. However, we do not know how the PCIDs should interact.

To facilitate fitting of the CSN1 and CSN7 structures into the envelope, we performed mutagenesis on CSN1. First, we constructed several recombinant atCSN1 C-terminal truncations for size-exclusion chromatography (SEC) comigration study (Fig. $4 B)$. As we anticipated, atCSN1 (16-400aa) with a complete 

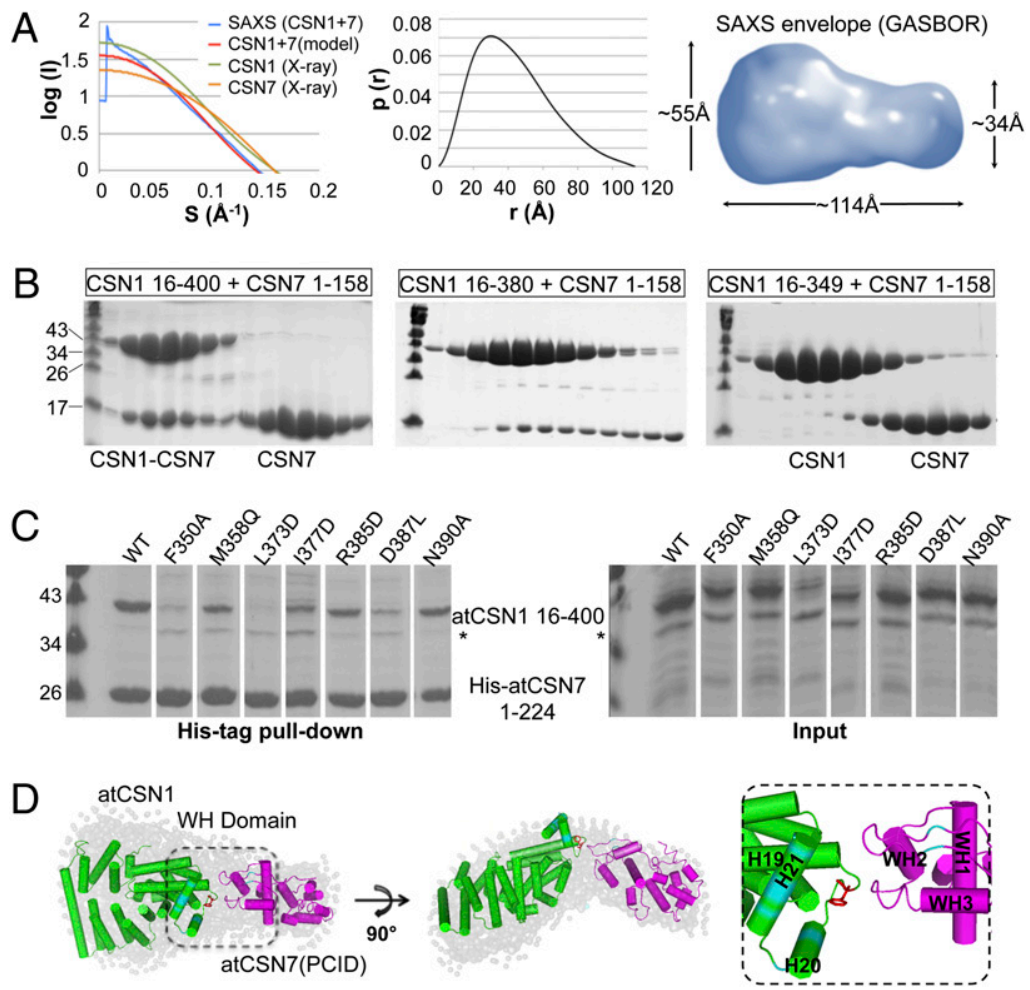

Fig. 4. atCSN1 interacts with atCSN7 (PCID) through its WH subdomain. (A) SAXS study on atCSN1-atCSN7 (PCID) binary complex. Scattering curve and distance distribution function of the binary complex are shown on the Left, and the SAXS envelope with dimensions is shown on the Right. (B) Size-exclusion chromatography (SEC) study between atCSN7 (PCID) and various atCSN1 constructs. (Left) atCSN7 (PCID) comigrated with atCSN1 (16-400aa), which contains a complete PCID. (Center) PCID-PCID interaction was affected but not eliminated when we truncated the last two $\beta$-strands in atCSN1. (Right) PCID-PCID interaction was abrogated when atCSN1 construct only had $\mathrm{H} 19$ in the $\mathrm{WH}$ subdomain. Residues from 350 to 400 of atCSN1 are essential for interaction with atCSN7. (C) Effects on atCSN7 interaction by mutations of conserved residues from 350 to 400 of atCSN1. (Right) Expression levels of atCSN1 wild-type/ mutation constructs and N-terminal His-tagged atCSN7. (Left) Pull-down experiments performed using Ni beads. (D) atCSN1 and atCSN7 (PCID) crystal structures (34) were fitted into the SAXS envelope. The SAXS fitting shows that the two molecules most likely interact through the WH subdomains (dashed line area). F350 (red) of atCSN1 is at the interface with atCSN7. Residues whose mutations did not affect the atCSN1-atCSN7 mutual interaction, including K144 and E153 of atCSN7 (34), are colored in cyan.

PCID was capable of interacting with atCSN7 (PCID), and the binary complex comigrated on SEC (Fig. $4 B$, Left). When we deleted the last two $\beta$-strands in the atCSN1 WH subdomain (16380aa), the PCID-PCID interaction was affected but not eliminated (Fig. 4B, Center). It was when we kept only H19 and removed everything else in the atCSN1 WH subdomain (16-349aa) that the interaction within the binary complex was completely abolished (Fig. 4B, Right). These results indicate that residues from 350 to 400 of atCSN1 containing the C-terminal part of the WH domain of atCSN1 are essential for interaction with atCSN7.

To further map which region of atCSN1 may directly mediate the interaction with atCSN7, we selected conserved residues from 350 to 400 and mutated those that are exposed or at least partly exposed to solvent (Figs. 2 and $4 C$ ). We included conserved residues beyond our atCSN1 structure to assess their role, if any, in atCSN7 interaction. Most prominently, the F350A mutation almost completely abolished the interaction with atCSN7, and D387L greatly weakened the interaction. L373D also abolished the interaction with atCSN7, but the structural integrity of the mutant may have been compromised as suggested by the much lower expression level. In contrast, mutations M358Q, I377D, R385D, and N390A did not greatly reduce the interaction with atCSN7. The mutagenesis data provided a guide for fitting of CSN1. In addition, it has been shown previously that mutations K144A and E153A in atCSN7 did not affect the interaction with atCSN1 (34); the data were also used to guide fitting. The resulting structural model predicted an interaction in which helices and the in-between loops of the WH subdomains of atCSN1 and atCSN7 are in contact with each other (Fig. 4D). The C-terminal $\beta$-strands of atCSN7 lie tangentially with the $\beta$-hairpin loop close to the interface. Such an orientation of the region may be taken by the C-terminal $\beta$-strands of atCSN1 as well in the structure with a complete PCID. The tangential location would explain the lack of strong effects when deleted in atCSN1; however, if a noncompatible mutation is introduced to a residue near the $\beta$-hairpin loop, such as D387L of atCSN1, interference with interaction could occur. Collectively, although the fitted model could not be atomically accurate, it provides a visual means for the possible mode of interaction between atCSN1 and atCSN7.

atCSN1 in the CSN-SCF Complex and the apo-CSN. CSN1 is known to play a crucial role in the integrity of the CSN complex $(37,39$ 41). To identify functionally important regions in CSN1, we mapped the sequence similarity score onto the surface of the atCSN1 crystal structure (Fig. 5A). Analysis shows that conserved areas lie at the surfaces of PCID and the LH and HR-II domains. A number of studies suggested that PCID mediates and stabilizes protein-protein interactions within the CSN complex (32-34). We specifically show that atCSN1 residues 350-400, a segment within the WH subdomain, are essential for interaction with atCSN7 (Fig. 4B). Our results, together with others mentioned, are consistent with the identified conserved PCID surface. In the EM structure of the CSN coupled with $\mathrm{SCF}, \mathrm{CSN} 1, \mathrm{CSN} 2, \mathrm{CSN} 3, \mathrm{CSN} 4$, and CSN7 form an approximately coplanar structure through the PCIDs (38). Based on this 

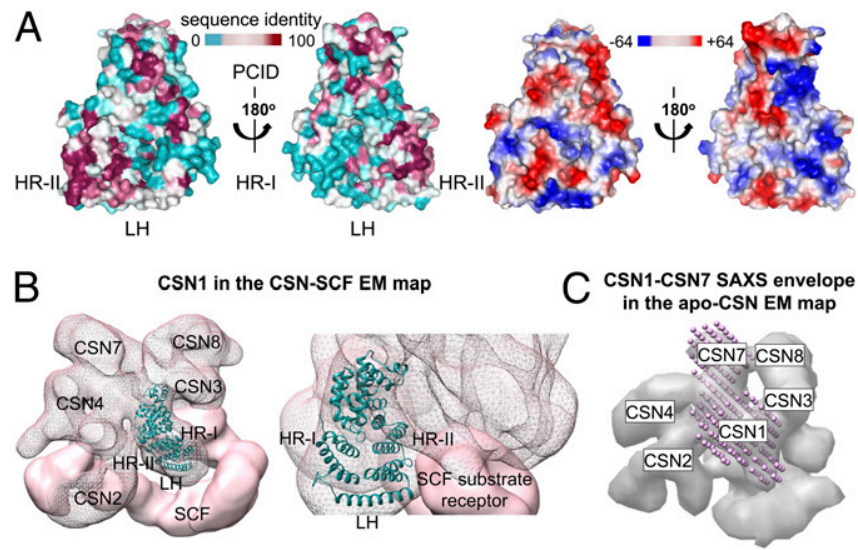

Fig. 5. atCSN1 in the EM maps of apo-CSN and the CSN-SCF Complex. $(A)$ Surface analysis of atCSN1 structure. (Left) Sequence similarity mapped onto the surface of atCSN1. Sequence alignment of CSN1 was first performed using different species; then the similarity score was mapped onto the surface of atCSN1. A cyan-white-magenta color gradient is used to represent the sequence similarity. (Right) Surface charge analysis on atCSN1. ( $B$, Left) atCSN1 structure was fitted into the CSN-SCF EM map (38). (Right) According to the surface analysis from $A$, the conserved regions within LH and HR-II of atCSN1 are interacting with the substrate receptor end of SCF. Conserved surface residues within the LH and HR-II are indicated using gray circles in Fig. 2. (C) SAXS envelope of the atCSN1-atCSN7 (PCID) binary complex from Fig. $4 A$ was fitted into the EM map of the apo-CSN complex (36).

arrangement, it is possible that different surfaces of the atCSN1 PCID are responsible for interacting with the neighboring CSN subunits. However, a detailed understanding on how the PCIDs interact with each other in the CSN complex has to wait for a high-resolution CSN complex structure.

Interestingly, the EM structure of the CSN-SCF complex suggested that the N-terminal domains of CSN1 and CSN3 are connected with the substrate-recognition end of the SCF (38). Therefore, we fitted the atCSN1 structure into this $25-\AA$ EM map using the program Chimera (44) to determine how CSN1 may reside within the CSN-SCF complex (Fig. 5B). The fitting generated a correlation coefficient, which indicates the agreement between the structure and the volume map, of 0.86 . The overall position of the fitted atCSN1 crystal structure in the CSN-SCF complex is comparable with the one shown using predicted atomic model for CSN1-volume docking (38), with the PCID of atCSN1 situating in some interior density of the CSN. Remarkably, the conserved regions in the LH and HR-II domains of atCSN1 are contacting the SCF substrate receptor according to our fitting (Fig. $5 B$ ).

During the fitting process, we noticed a difference in the assigned CSN1-CSN7 volumes between the CSN-SCF EM map and the apo-CSN EM map $(36,38)$. In the CSN-SCF EM map, CSN1 and CSN7 do not directly contact each other with a gap in the central arc region of the approximate coplanar arrangement (38). However, in the apo-CSN EM map, this gap does not exist, with apparent continuous densities between CSN1 and CSN7. To confirm this observation, we docked our atCSN1-atCSN7 (PCID) SAXS envelope into the apo-CSN EM map. As predicted, the SAXS envelope aligned well with the assigned densities of the CSN1-CSN7 volume (Fig. 5C). One possible explanation is that the CSN experiences subunit rearrangements upon association with SCF, a plasticity that is supported by several additional lines of evidence. First, in vitro, CSN7 can interact with either CSN1 or CSN8 to form binary complexes, but not simultaneously to form ternary complexes (34). Second, in the apo-CSN, although CSN7 clearly interacts with CSN1, it barely touches CSN8 (36). Third, in the CSN-SCF complex, CSN7 contacts neither CSN1 nor CSN8
(38). Collectively, these data support conformational variability in the PCID interactions in the CSN.

atCSN1 Interacts with IкB $\alpha$ Through Its C-terminal Tail. So far, we have confirmed that the PCID of atCSN1 is important for PCIDPCID interactions whereas the HR-II and LH domains may occlude the substrate-recognition end of the SCF. The N-terminal half of CSN1 was reported to interact with Tonsoku-associating protein 1 (45), SAP130/SF3b-3 (46), inositol 1,3,4-trisphosphate 5/6-kinase (17), and ankyrin repeat and SOCS box-containing protein 4 (47). Here, we report that the C-terminal tail beyond the PCID of $H$. sapiens CSN1 (hsCSN1), a segment not included in our crystal structure, interacts with $\mathrm{I} \kappa \mathrm{B} \alpha$ in the $\mathrm{NF}-\kappa \mathrm{B}$ signaling pathway. We first made a c-Myc-tagged $\mathrm{I} \kappa \mathrm{B} \alpha$ and various Flagtagged hsCSN constructs, hsCSN1-8 (full length) and hsCSN1 truncation mutants (1-461aa) and (1-362aa). The proteins were in vitro translated one at a time in a cell-free environment. The translated sample containing expressed I $\mathrm{KB} \alpha$ was incubated with each CSN-containing in vitro translated sample. After immunoprecipitation of Myc-IкB $\alpha$ from the different samples, interactions between the individual CSN subunit and IкB $\alpha$ were detected using an anti-Flag antibody (Fig. 6A, Fig. S4). Without the presence of other CSN subunits in the in vitro translation solutions, hsCSN1 interacted with I $\mathrm{KB} \alpha$ through residues 461527. According to secondary structure prediction, this region contains an $\sim 20$-aa $\alpha$-helix followed by some disordered segments. The C-terminal tail of hsCSN1 is most likely flexible as we do not visualize extra density around the CSN1 (PCID) region in either the CSN or the CSN-SCF EM map. The flexible tail is then possibly exposed for interaction with I $\mathrm{B} \alpha$ in the context of the full CSN (Fig. 6B). The only other subunit that interacted with
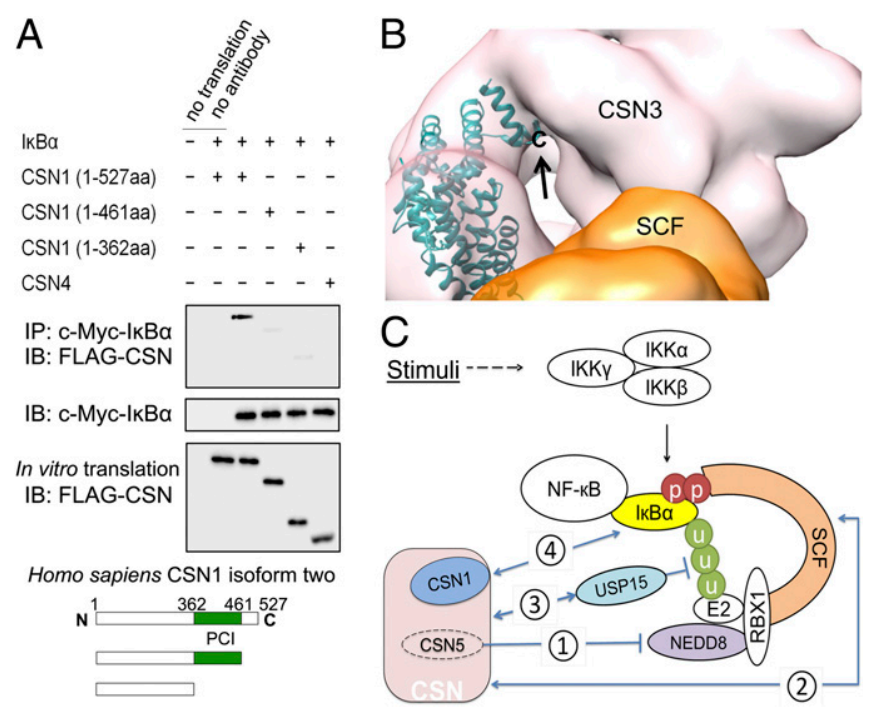

Fig. 6. CSN1 interacts with $1 \kappa B \alpha$ through its C-terminal tail. (A) Pull-down results between in vitro translated hsCSN1 and IкB $\alpha$. C-Myc-tagged $I_{\kappa} \mathrm{B} \alpha$ and four Flag-tagged $H$. sapiens (hs) CSN constructs, hsCSN1 (full length), hsCSN1 (1-461aa), hsCSN (1-362aa), and hsCSN4 (full length), were constructed. Lane 3 shows that CSN1 interacts with $1 \kappa B \alpha$ through residues $461-527$. (B) The flexible tail of CSN1 is possibly exposed for interaction with $I_{\kappa} B \alpha$ in the context of the full CSN. atCSN1 in the EM map of the CSN-SCF complex, and the arrow indicates the C-terminal end of our atCSN1 structure. (C) CSN complex in NF-KB pathways. One, CSN complex influences protein stability by removing Nedd8 from cullin-RING-E3 ligase $(9,14)$. Two, CSN complex can inhibit a catalytically competent SCF by binding both substrate receptor and Rbx1-Cul1 C-terminal domain of SCF complex (38). Three, CSN complex is known to interact with deubiquitylation enzymes USP15 to suppress NF- $\mathrm{kB}$ activation $(12,22)$. Four, the C-terminal tail of CSN1 interacts with $1 \kappa B \alpha$ with current unknown mechanism. IB, immunoblotting; IP, immunoprecipitation. 
IкB $\alpha$ is CSN3 (Fig. S4), suggesting that the adjacently located CSN1 and CSN3 cooperate to recruit IкB $\alpha$.

In NF-kB pathways, as one example, the CSN complex acts as a platform arranging protein communications (Fig. 6C). Upon

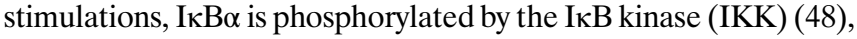
which is then recognized by the substrate receptor of the SCF for polyubiquitination, leading to subsequent proteasomal degradation. Degradation of IкB $\alpha$ releases NF-kB for nuclear translocation and activation of transcription. Four, or more, direct interactions between the CSN and the players within the pathway may lead to regulation of NF-kB activities. One, by removing Nedd8 from the SCF E3 ligase, the CSN complex impoverishes the assembly and ubiquitination activity of the SCF complex $(49,50)$. Thus, deneddylation here leads to NF-kB inactivation. Two, the CSN complex can also inhibit a catalytically competent SCF by directly interacting with both substrate receptor and Rbx1-Cul1 Cterminal domain of the SCF complex (38). Three, the CSN complex is known to interact with the deubiquitination enzyme USP15 to suppress NF-kB activation $(12,22)$. Four, we show that the C-terminal tail of CSN1 interacts with $\mathrm{I} \kappa \mathrm{B} \alpha$, possibly bringing

1. Wei N, Deng XW (1992) COP9: A new genetic locus involved in light-regulated development and gene expression in arabidopsis. Plant Cell 4(12):1507-1518.

2. Chamovitz DA, et al. (1996) The COP9 complex, a novel multisubunit nuclear regulator involved in light control of a plant developmental switch. Cell 86(1):115-121.

3. Mundt KE, et al. (1999) The COP9/signalosome complex is conserved in fission yeast and has a role in S phase. Curr Biol 9(23):1427-1430.

4. Wee S, Hetfeld B, Dubiel W, Wolf DA (2002) Conservation of the COP9/signalosome in budding yeast. $B M C$ Genet 3:15.

5. Luke-Glaser S, et al. (2007) CIF-1, a shared subunit of the COP9/signalosome and eukaryotic initiation factor 3 complexes, regulates MEL-26 levels in the Caenorhabditis elegans embryo. Mol Cell Biol 27(12):4526-4540.

6. Freilich S, et al. (1999) The COP9 signalosome is essential for development of Drosophila melanogaster. Curr Biol 9(20):1187-1190.

7. Seeger $M$, et al. (1998) A novel protein complex involved in signal transduction possessing similarities to $26 \mathrm{~S}$ proteasome subunits. FASEB J 12(6):469-478.

8. Wei N, Deng XW (1998) Characterization and purification of the mammalian COPS complex, a conserved nuclear regulator initially identified as a repressor of photomorphogenesis in higher plants. Photochem Photobiol 68(2):237-241.

9. Lyapina S, et al. (2001) Promotion of NEDD-CUL1 conjugate cleavage by COP9 signalosome. Science 292(5520):1382-1385.

10. Schwechheimer $C$, et al. (2001) Interactions of the COP9 signalosome with the E3 ubiquitin ligase SCFTIRI in mediating auxin response. Science 292(5520):1379-1382.

11. Zhou C, et al. (2001) The fission yeast COP9/signalosome is involved in cullin modification by ubiquitin-related Ned8p. BMC Biochem 2:7.

12. Hetfeld BK, et al. (2005) The zinc finger of the CSN-associated deubiquitinating enzyme USP15 is essential to rescue the E3 ligase Rbx1. Curr Biol 15(13):1217-1221.

13. Zhou C, et al. (2003) Fission yeast COP9/signalosome suppresses cullin activity through recruitment of the deubiquitylating enzyme Ubp12p. Mol Cell 11(4):927-938.

14. Cope GA, et al. (2002) Role of predicted metalloprotease motif of Jab1/Csn5 in cleavage of Nedd8 from Cul1. Science 298(5593):608-611.

15. Naumann M, Bech-Otschir D, Huang X, Ferrell K, Dubiel W (1999) COP9 signalosomedirected c-Jun activation/stabilization is independent of JNK. J Biol Chem 274(50): 35297-35300.

16. Uhle $S$, et al. (2003) Protein kinase CK2 and protein kinase D are associated with the COP9 signalosome. EMBO J 22(6):1302-1312.

17. Sun Y, Wilson MP, Majerus PW (2002) Inositol 1,3,4-trisphosphate 5/6-kinase associates with the COP9 signalosome by binding to CSN1. J Biol Chem 277(48):45759-45764.

18. Bech-Otschir D, et al. (2001) COP9 signalosome-specific phosphorylation targets p53 to degradation by the ubiquitin system. EMBO J 20(7):1630-1639.

19. Tomoda K, et al. (2002) The cytoplasmic shuttling and subsequent degradation of p27Kip1 mediated by Jab1/CSN5 and the COP9 signalosome complex. J Biol Chem 277(3):2302-2310

20. Wang $X$, et al. (2009) Regulation of COP1 nuclear localization by the COP9 signalosome via direct interaction with CSN1. Plant J 58(4):655-667.

21. Groisman R, et al. (2003) The ubiquitin ligase activity in the DDB2 and CSA complexes is differentially regulated by the COP9 signalosome in response to DNA damage. Cell 113(3):357-367.

22. Schweitzer K, Bozko PM, Dubiel W, Naumann M (2007) CSN controls NF-kappaB by deubiquitinylation of IkappaBalpha. EMBO J 26(6):1532-1541.

23. Lykke-Andersen K, et al. (2003) Disruption of the COP9 signalosome Csn2 subunit in mice causes deficient cell proliferation, accumulation of p53 and cyclin $\mathrm{E}$, and early embryonic death. Mol Cell Biol 23(19):6790-6797.

24. Dohmann EM, et al. (2008) The Arabidopsis COP9 signalosome is essential for G2 phase progression and genomic stability. Development 135(11):2013-2022.

25. Glickman MH, Rubin DM, Fried VA, Finley D (1998) The regulatory particle of the Saccharomyces cerevisiae proteasome. Mol Cell Biol 18(6):3149-3162.
IкB $\alpha$ to CSN-recruited USP15 for deubiquitination. Such a recruiting mechanism may be facilitated by the neighboring CSN3 because we learned that IкB $\alpha$ directly interacts with either CSN1 or CSN3 (Fig. S4). Overall, the CSN complex uses multiple mechanisms to hinder NF-kB activation, a principle likely to hold true for its regulation of many other targets and pathways.

\section{Materials and Methods}

atCSN1 was expressed in E. coli BL21 (DE3). All proteins were purified using $\mathrm{Ni}$-affinity chromatography followed by anion-exchange chromatography (Uno-Q) and SEC (16/60 Superdex-200pg). atCSN1 (16-400aa) was crystallized at $20^{\circ} \mathrm{C}$ in $0.1 \mathrm{M}$ Bis-tris propane, $\mathrm{pH} 7.0,0.2 \mathrm{M}$ ammonium sulfate, $10 \%$ (wt/ vol) polyethylene glycol 8000 , and $5 \mathrm{mM}$ DTT. The structure was determined by the multi-wavelength anomalous diffraction (MAD) method using a $\left[\mathrm{Ta}_{6} \mathrm{Br}_{12}\right]^{2+}$ soaked crystal.

For detailed experimental procedures, please see SI Materials and Methods.

ACKNOWLEDGMENTS. CDNAs of $A$. thaliana CSN subunits were kindly provided by Professor Xing-Wang Deng (Yale University). In addition, we thank Dr. Tianmin Fu, Dr. Devendra Srivastava, and Alvin Lu for valuable discussions.

26. Maytal-Kivity V, Reis N, Hofmann K, Glickman MH (2002) MPN+, a putative catalytic motif found in a subset of MPN domain proteins from eukaryotes and prokaryotes, is critical for Rpn11 function. BMC Biochem 3:28

27. Wolf DA, Zhou C, Wee S (2003) The COP9 signalosome: An assembly and maintenance platform for cullin ubiquitin ligases? Nat Cell Bio/ 5(12):1029-1033.

28. Zhang $\mathrm{H}$, et al. (2012) The crystal structure of the MPN domain from the COP9 signalosome subunit CSN6. FEBS Lett 586(8):1147-1153.

29. Echalier A, et al. (2013) Insights into the regulation of the human COP9 signalosome catalytic subunit, CSN5/Jab1. Proc Natl Acad Sci USA 110(4):1273-1278.

30. Hofmann K, Bucher P (1998) The PCl domain: A common theme in three multiprotein complexes. Trends Biochem Sci 23(6):204-205

31. Scheel H, Hofmann K (2005) Prediction of a common structural scaffold for proteasome lid, COP9-signalosome and elF3 complexes. BMC Bioinformatics 6:71.

32. Fu H, Reis N, Lee Y, Glickman MH, Vierstra RD (2001) Subunit interaction maps for the regulatory particle of the $26 \mathrm{~S}$ proteasome and the COP9 signalosome. EMBO J 20(24): 7096-7107.

33. Serino G, et al. (2003) Characterization of the last subunit of the Arabidopsis COP9 signalosome: Implications for the overall structure and origin of the complex. Plant Cell 15(3):719-731.

34. Dessau M, et al. (2008) The Arabidopsis COP9 signalosome subunit 7 is a model PCI domain protein with subdomains involved in COP9 signalosome assembly. Plant Cell 20(10):2815-2834.

35. Kapelari B, et al. (2000) Electron microscopy and subunit-subunit interaction studies reveal a first architecture of COP9 signalosome. J Mol Biol 300(5):1169-1178.

36. Enchev RI, Schreiber A, Beuron F, Morris EP (2010) Structural insights into the COP9 signalosome and its common architecture with the $26 \mathrm{~S}$ proteasome lid and elF3. Structure 18(4):518-527.

37. Sharon M, et al. (2009) Symmetrical modularity of the COP9 signalosome complex suggests its multifunctionality. Structure 17(1):31-40.

38. Enchev RI, et al. (2012) Structural basis for a reciprocal regulation between SCF and CSN. Cell Rep 2(3):616-627.

39. Tsuge $T$, Matsui $M$, Wei $N$ (2001) The subunit 1 of the COP9 signalosome suppresses gene expression through its $\mathrm{N}$-terminal domain and incorporates into the complex through the PCl domain. J Mol Biol 305(1):1-9.

40. Wang Y, Devereux W, Stewart TM, Casero RA, Jr. (2002) Polyamine-modulated factor 1 binds to the human homologue of the 7a subunit of the Arabidopsis COP9 signalosome: Implications in gene expression. Biochem J 366(Pt 1):79-86.

41. Peth A, Berndt C, Henke W, Dubiel W (2007) Downregulation of COP9 signalosome subunits differentially affects the CSN complex and target protein stability. BMC Biochem 8:27.

42. Zeytuni N, Zarivach R (2012) Structural and functional discussion of the tetra-tricopeptide repeat, a protein interaction module. Structure 20(3):397-405.

43. Pathare GR, et al. (2012) The proteasomal subunit Rpn6 is a molecular clamp holding the core and regulatory subcomplexes together. Proc Natl Acad Sci USA 109(1):149-154.

44. Pettersen EF, et al. (2004) UCSF Chimera-a visualization system for exploratory research and analysis. J Comput Chem 25(13):1605-1612.

45. Li W, et al. (2011) TSA1 interacts with CSN1/CSN and may be functionally involved in Arabidopsis seedling development in darkness. J Genet Genomics 38(11):539-546.

46. Menon S, Tsuge T, Dohmae N, Takio K, Wei N (2008) Association of SAP130/SF3b-3 with Cullin-RING ubiquitin ligase complexes and its regulation by the COP9 signalosome. BMC Biochem 9:1.

47. Li JY, et al. (2007) Ankyrin repeat and SOCS box containing protein 4 (Asb-4) interacts with GPS1 (CSN1) and inhibits C-Jun NH2-terminal kinase activity. Cell Signal 19(6): $1185-1192$.

48. Xu G, et al. (2011) Crystal structure of inhibitor of $\kappa B$ kinase $\beta$. Nature 472(7343):325-330

49. Saha A, Deshaies RJ (2008) Multimodal activation of the ubiquitin ligase SCF by Nedd8 conjugation. Mol Cell 32(1):21-31.

50. Duda DM, et al. (2008) Structural insights into NEDD8 activation of cullin-RING ligases: Conformational control of conjugation. Cell 134(6):995-1006. 